\title{
Priorización Multicriterio de Mercados Potenciales de Comercio Justo
}

\author{
Sebastián C. Araya-Pizarro(1)* y Camilo R. Araya-Pizarro(2) \\ (1) Facultad de Cs. Sociales y Económicas, Dpto. de Cs. Económicas y Empresariales, Universidad de La Serena, \\ Amunátegui s/n, La Serena-Chile (e-mail: saraya@userena.cl) \\ (2) Facultad de Economía y Negocios, Universidad Santo Tomás, Ruta 5 Norte s/n, La Serena-Chile \\ (e-mail: carayap@santotomas.cl) \\ * Autor a quien debe ser dirigida la correspondencia
}

Recibido Dic. 26, 2018; Aceptado Feb. 28, 2019; Versión final Abr. 5, 2019, Publicado Oct. 2019

\begin{abstract}
Resumen
El artículo evalúa los potenciales mercados internacionales de Comercio Justo para la Región de Coquimbo (Chile) a través de la metodología de toma de decisiones multicriterio denominada Proceso Analítico Jerárquico (Analytic Hierarchy Process, AHP). La estructura jerárquica del modelo analizó dos grupos de criterios de valoración: potencial del mercado (crecimiento económico, poder adquisitivo, volumen importaciones, crecimiento de las importaciones y exportaciones) y accesibilidad y riesgo (libertad económica, riesgo país, facilidad para hacer negocios, transparencia y corrupción), en 28 países con Comercio Justo. Los resultados indican que los mercados con mayor prioridad están en América del Norte (USA, Canadá), Europa (Noruega, Dinamarca, Alemania y Suiza) y Oceanía (Nueva Zelanda y Australia). Como conclusión destaca la fortaleza del Método AHP para la priorización de mercados y la posibilidad de replicarlo en contextos afines. Cabe esperar que estos hallazgos constituyan el primer paso para el diseño exitoso de estrategias de exportación en base a Comercio Justo.
\end{abstract}

Palabras clave: comercio justo; proceso analítico jerárquico (AHP); mercados potenciales; mercados internacionales

\section{Multicriterial Prioritization of Potential Fair Trade Markets}

\begin{abstract}
The article evaluates the potential international markets of Fair Trade for the Region of Coquimbo (Chile) through the multicriteria decision making methodology known as Analytic Hierarchy Process (AHP). The hierarchical structure of the model analyzed two groups of valuation criteria: market potential (economic growth, purchasing power, volume imports, growth of imports and exports) and accessibility and risk (economic freedom, country risk, ease of doing business, transparency and corruption), in 28 countries with Fair Trade. The results indicate that the markets with the highest priority are in North America (USA, Canada), Europe (Norway, Denmark, Germany and Switzerland) and Oceania (New Zealand and Australia). In conclusion, it highlights the strength of the AHP Method for prioritizing markets and the possibility of replicating it in related contexts. It is hoped that these findings will be the first step in the successful design of export strategies based on Fair Trade.
\end{abstract}

Keywords: fair trade; analytic hierarchy process (AHP); potential markets; international market 


\section{INTRODUCCIÓN}

Producir y consumir sobre la base de principios éticos y responsables son comportamientos de creciente interés social (Ulusoy, 2016). La sociedad civil y especialmente algunas organizaciones no gubernamentales han desarrollado una aproximación propia a la dinámica del consumo responsable. En esta corriente se propone la sensibilización social sobre el papel del consumo como comportamiento humano en un mercado globalizado (Llopis-Going, 2009). Con ello se busca concienciar sobre la necesidad de exigir los derechos ya consolidados del consumidor a optar por productos que cumplan criterios "socialmente éticos" o "socialmente responsables", como por ejemplo: rechazo al trabajo infantil, igualdad de salarios, respeto medioambiental, trato adecuado a los trabajadores, apoyo a programas de lucha contra la pobreza, entre otras acciones (Marquina y Reficco, 2015).

El Comercio Justo (o Fair Trade) es un sistema comercial alternativo que busca alcanzar mayor equidad en el comercio internacional mediante el establecimiento de mejores condiciones comerciales que garantizan los derechos de los productores y trabajadores marginados (Garza, 2014). Tiene como objetivo principal disminuir las condiciones de marginación y pobreza en las que viven millones de campesinos y pequeños productores provenientes de países en desarrollo (Chávez y Patraca, 2011), promoviendo así un mundo más justo y sostenible basado en la cooperación y la transparencia. Por todo lo antes mencionado, el Comercio Justo es considerado una herramienta de cooperación de largo plazo y una iniciativa popular alterna para regular socialmente los mercados globales, particularmente en el sector agroalimentario (Raynolds, 2012).

El proceso del Comercio Justo se enmarca en el cumplimiento de diez principios internacionales reguladores: (i) Creación de oportunidades para productores con desventajas económicas; (ii) Transparencia y responsabilidad; (iii) Prácticas comerciales justas; (iv) Pago de un precio justo; (v) Asegurar ausencia de trabajo infantil y trabajo forzoso; (vi) Compromiso con la no discriminación, equidad de género y libertad de asociación; (vii) Asegurar buenas condiciones de trabajo; (viii) Facilitar el desarrollo de capacidades; (ix) Promoción del Comercio Justo; y (x) Respeto por el medio ambiente. Estos criterios se traducen en una serie de beneficios conexos para los productores, entre los que se cuentan: el sobreprecio, la prefinanciación, las relaciones a largo plazo, la seguridad y estabilidad potencial de participar de las redes de Comercio Justo, lo cual también podría reflejarse en el bienestar de las comunidades en las que viven. De allí que muchos pequeños productos de América Latina han decidido participar del sistema. Al respecto, Hopkins (2000), en su estudio sobre la influencia del Comercio Justo en las condiciones de vida de varios productores artesanales en el mundo (Perú, Salvador, Guatemala, India, Filipinas, Bangladesh y Ghana), reveló que, a largo plazo, el Comercio Justo genera un incremento de ingresos, un aumento de las capacidades de los grupos y consiguientemente una mejoría en las condiciones de vida de la comunidad. Cabe mencionar que los productores de Comercio Justo garantizan que sus procesos de producción cumplen con los criterios establecidos, al ser certificados por entidades avaladas por la Organización Mundial del Comercio Justo, y que son las encargadas de otorgar el sello Comercio Justo (Fairtrade International, Ecocert, Fundeppo, IMOfair for life y Naturland).

En general, el Comercio Justo busca resolver dos grandes desafíos: Primero, lograr un mayor equilibrio del comercio mundial en beneficio de los actores más desfavorecidos, generando un impacto positivo dentro de las economías locales. En segundo lugar, un efecto demostrativo, al instaurar una realidad práctica de que es posible otro comercio, uno que logre concienciar a los consumidores y empresarios sobre una opción distinta de enfrentar las relaciones comerciales en el ámbito nacional e internacional (Comet, 2016). En Chile, actualmente, existen diversas organizaciones de productores y empresas con una gran variedad de productos en Comercio Justo, además de algunas redes que desarrollan interesantes iniciativas productivas y comerciales enfocadas en los mercados locales, nacionales e internacionales con una incidencia social y económica relevante. Todo ello favorecido por un contexto de mayor desarrollo del país y de un creciente número de consumidores más conscientes y responsables (Proqualitas, 2015).

En particular, la IV Región de Coquimbo presenta una matriz productiva de gran potencial agroexportador para países importadores de Comercio Justo, en especial para los sectores primarios de exportación de fruta fresca y el sector agroindustrial. La Región dispone de aproximadamente 76.000 hectáreas de superficie agroalimentaria bajo riego, destacando frutos como el almendro, arándano, cerezo, damasco, limonero, mandarines, manzanos, nogal, olivo, palto, peral y vid, además destaca el $94,7 \%$ de la superficie chilena de producción de uva pisquera. También existen importantes extensiones en especies como el chirimoyo, granada y papaya (Yañez, 2018).

El presente estudio tiene por objetivo priorizar los principales mercados internacionales demandantes de productos agroalimentarios Comercio Justo para la IV Región, mediante la utilización del Proceso Analítico Jerárquico (AHP - Analytic Hierarchy Process). La programación multicriterio con el proceso AHP está estructurada para la toma de decisiones en entornos complejos, en los que se consideran varias variables 0 
criterios para priorizar y seleccionar alternativas (Wollmann et al., 2014). El AHP ayuda a establecer el nivel de importancia entre los criterios y subcriterios definidos para la selección, incorporando tanto aspectos cualitativos como cuantitativos del pensamiento humano: los cualitativos para describir el problema y su jerarquía, y los cuantitativos para expresar los juicios y preferencias de manera concisa. Considera la consistencia de los juicios emitidos y además tiene soporte de software para hacer los cálculos y el análisis de sensibilidad (Brunelli, 2015; Oddershede et al., 2007; Saaty y Vargas, 2012).

Se espera que los resultados del estudio permitan a los productores certificados con Comercio Justo diseñar estrategias de comercialización efectivas enfocadas en la atención de mercados internacionales prioritarios, es decir, con potencial de mercado y condiciones favorables en cuanto a accesibilidad y riesgo.

\section{METODOLOGÍA}

El estudio se desarrolló bajo un paradigma positivista, de enfoque cuantitativo, diseño no experimental, de tipo descriptivo y corte transversal, en el que mediante el uso de la metodología AHP se examinaron dos grupos de criterios de valoración para la selección y/o priorización de los mercados internacionales: el potencial del mercado (crecimiento económico, poder adquisitivo, volumen importaciones, crecimiento de las importaciones y exportaciones) y la accesibilidad \& riesgo (libertad económica, riesgo país, facilidad para hacer negocios, transparencia y corrupción), en 28 países con Comercio Justo en el mundo.

El método AHP es un método lógico y estructurado, utilizado para optimizar la toma de decisiones complejas cuando existen múltiples criterios o atributos, a través de la descomposición del problema en una estructura jerárquica (Osorio et al., 2017). Esto permite subdividir un atributo complejo en un grupo de atributos más sencillos y determinar cómo influyen cada uno de esos atributos individuales en el objetivo decisional. De este modo, la selección del método AHP para la priorización multicriterio de mercados potenciales de Comercio Justo se justifica en el potencial que brinda para evaluar diferentes alternativas de mercados de exportación, en base al conjunto de criterios y subcriterios propuestos, en donde la opinión de los expertos consultados (importadores de Comercio Justo, productores certificados Comercio Justo, especialistas en comercio internacional y encargados de la promoción de la producción/exportación en Chile) se estima tan importante como los propios datos usados en el proceso.

Los pasos básicos del método AHP son los cuatro siguientes: i) Creación de una estructura jerárquica; ii) Creación de matriz de comparación; iii) Cálculo de prioridades; y iv) Cálculo del índice de consistencia y ratio.

Creación de una estructura jerárquica: La jerarquía se compone de diferentes niveles, representados por el objetivo de la toma de decisiones (nivel más alto), los criterios de decisión (nivel intermedio), y las alternativas de decisión (nivel más bajo).

Creación de matriz de comparación: La matriz de comparación de elementos en un nivel en relación con los elementos en un nivel superior se construye utilizando comparaciones individuales traducidas a valores de escala. Las preferencias de los tomadores de decisiones se cuantifican utilizando la escala de nueve puntos de Saaty, con el siguiente significado: $1=$ igualmente importante, $3=$ ligeramente importante, $5=$ muy importante, 7 = mucho más importante, $9=$ extremadamente más importante. Los 2, 4, 6 y 8 representan los valores intermedios.

Cálculo de prioridades: La comparación por pares genera una matriz de las clasificaciones relativas para cada nivel de la jerarquía. El número de la matriz depende del número de elementos en cada nivel. Una vez creadas todas las matrices, se calcula el vector del peso relativo y máximo valor propio $\left(\lambda_{\max }\right)$ para cada matriz.

Cálculo del índice de consistencia y ratio: La validez de las comparaciones se puede evaluar a través de la relación de consistencia. Antes de calcular la relación de consistencia, es necesario calcular un índice de consistencia (IC) de una matriz $n x n$, que se define como la relación $\mathrm{Cl}=\left(\lambda_{\max }-n\right) /(n-1)$. El valor $\lambda_{\max }$ es el máximo valor propio de la matriz, y $\mathrm{n}$ es la dimensión de la matriz. El índice de consistencia (CR) se calcula como $\mathrm{CR}=\mathrm{Cl} / \mathrm{RI}$, donde el valor de RI es el índice de consistencia aleatorio. La Tabla 1 muestra los valores para el RI de las dimensiones de la matriz de 1-10.

Tabla 1: Índices aleatorios por tamaño de matriz

\begin{tabular}{|l|c|c|c|c|c|c|c|c|c|}
\hline Tamaño de la matriz $(\mathrm{N})$ & 2 & 3 & 4 & 5 & 6 & 7 & 8 & 9 & 10 \\
\hline Índice aleatorio $(\mathrm{RI})$ & 0 & 0,58 & 0,9 & 1,12 & 1,24 & 1,32 & 1,41 & 1,45 & 1,49 \\
\hline
\end{tabular}

El valor aceptable de la CR depende del tamaño de la matriz $(0,1$ para matrices $n \geq 5)$. Si el valor de $C R$ es igual o menor que el valor especificado, esto indica que la evaluación dentro de la matriz es aceptable y 
cercana a los valores ideales. Sin embargo, si el CR es más alto que el valor aceptable, el proceso de evaluación debe ser mejorado, lo que se logra en una reunión conjunta de los participantes de la investigación (Jovanović et al., 2015). La priorización multicriterio de los mercados potenciales de Comercio Justo, mediante la metodología AHP, siguió el esquema presentado en la Figura 1.

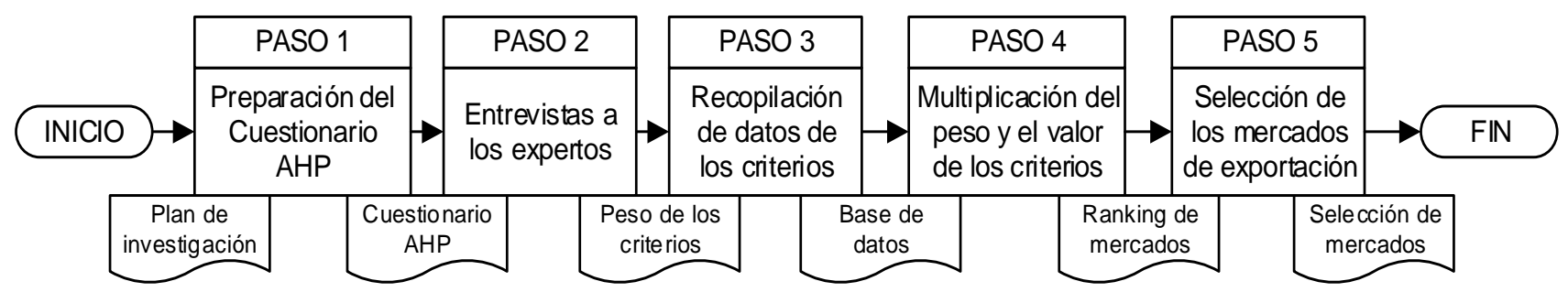

Fig. 1: El algoritmo del método AHP aplicado al estudio

Para la determinación de las variables consideradas en el modelo (criterios y subcriterios), se llevó a cabo una revisión bibliográfica preliminar que concluyó con la opinión de doce expertos. Específicamente se aplicaron entrevistas individuales estructuradas a tres autoridades de la Región de Coquimbo (secretario regional ministerial de economía, director de ProChile y director de la Corporación de Fomento de la Producción), a tres académicos expertos en comercio internacional (todos con posgrados afines), a tres importadores de Comercio Justo (Secretaria regional WFTO Latinoamérica, importador Fair Trade Holanda, Traidcraft Reino Unido) y a tres empresarios locales certificados bajo Comercio Justo (Cooperativa Agrícola Pisquera Elqui, Cooperativa Frutícola Agronuez Choapa y Pisco MalPaso). Los datos recopilados fueron examinados mediante el análisis de contenido de tipo categórico descriptivo, que permitió la identificación de nueve factores de interés. Las variables consideradas en el estudio se exponen en la Tabla 2.

Tabla 2: Variables para la priorización de los mercados internacionales

\begin{tabular}{|l|l|}
\hline Variable & Definición \\
\hline Crecimiento Económico & Variación del Producto Interno Bruto (PIB) para el periodo 2016-2015 (\%) \\
\hline Poder adquisitivo & PIB Per cápita en dólares, año 2016 (PPA US\$) \\
\hline Volumen importaciones & Importaciones en miles de millones de dólares, año 2016 (US\$) \\
\hline Crecimiento de las importaciones & Crecimiento promedio de las importaciones para el periodo 2012-2016 (\%) \\
\hline Exportaciones & Exportaciones en millones de dólares, año 2016 (US\$) \\
\hline Barreras al comercio & Índice de Libertad Económica, año 2016 \\
\hline Riesgo & Riesgo país 2016, año 2016 \\
\hline Facilidad para hacer negocios & Índice de facilidad para hacer negocios, año 2016 \\
\hline Transparencia y corrupción & Índice de Transparencia y corrupción, año 2016 \\
\hline
\end{tabular}

Luego, a través del método AHP se procedió a integrar, en un modelo de priorización, las variables antes mencionadas. Estas se denominaron subcriterios, las que fueron agrupadas en las dimensiones: potencial de mercado y accesibilidad \& riesgo, según la relación existente entre ellos. Es significativo precisar que en la dimensión accesibilidad \& riesgo, los 4 subcriterios de estudio corresponden a índices compuestos por subfactores, lo que permite contar con la riqueza que genera una perspectiva multivariada. Así, por ejemplo, solo en el caso del Índice de Libertad Económica, el indicador incluye 12 factores (derechos de propiedad, integridad del gobierno, efectividad judicial, gasto gubernamental, carga impositiva, saluda fiscal, libertad de empresa, libertad de trabajo, libertad monetaria, libertad de intercambio, libertad de inversión y libertad financiera) que permiten medir los efectos sobre 4 categorías de análisis (Estado de derecho, tamaño del gobierno, eficiencia regulatoria y apertura de mercado). A su vez el índice de facilidad para hacer negocios se obtiene por medio de 10 categorías, entre las que se cuentan aspectos como: apertura de un negocio, obtención de crédito, protección de los inversores, pago de impuestos y resolución de la insolvencia.

De esta forma, se generó una estructura jerárquica de 2 dimensiones compuesta de 5 subcriterios para la valoración del potencial de mercado (crecimiento económico, poder adquisitivo, volumen de importaciones, crecimiento de las importaciones y volumen de exportaciones), 4 subcriterios para la valoración de accesibilidad y riesgo (barreras al comercio, riesgo, facilidad para hacer negocios y transparencia \& corrupción) y 28 alternativas de mercado (Alemania, Australia, Austria, Bélgica, Brasil, Canadá, Corea del Sur, Dinamarca, Eslovaquia, España, Estados Unidos, Estonia, Finlandia, Francia, Holanda, Hong Kong, Irlanda, Italia, Letonia, Lituania, Luxemburgo, Noruega, Nueva Zelanda, Portugal, Reino Unido, República Checa, Suecia y Suiza), tal como se ilustra en la Figura 2. 


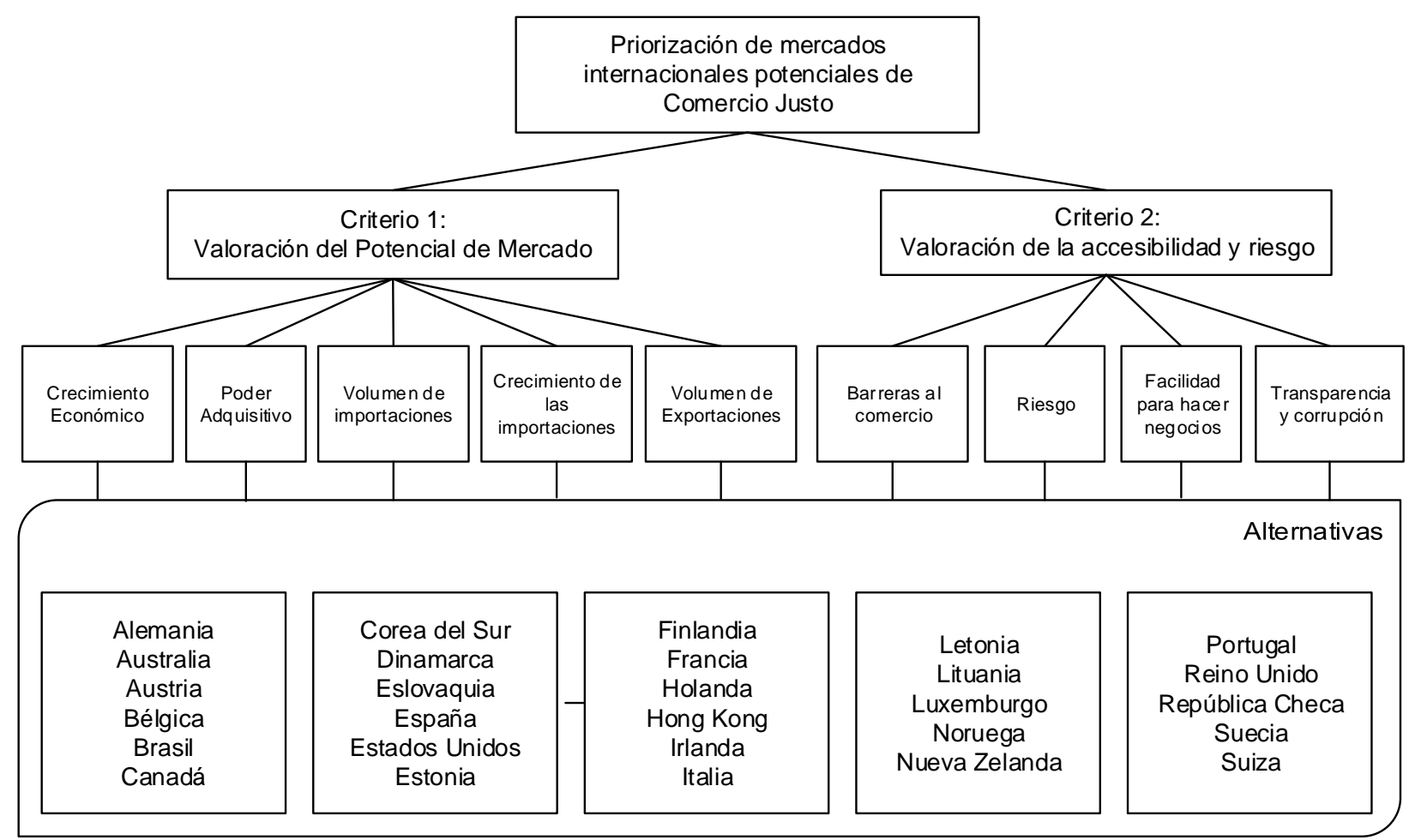

Fig. 2: Estructura jerárquica de criterios de valoración y de las alternativas de mercado

Cabe destacar que las alternativas de mercado elegidas para el análisis AHP corresponden a los principales países compradores de Comercio Justo del mundo (Véase la Tabla 3), y por tanto su selección asegura la presencia del segmento objetivo de estudio (consumidor responsable), que según Cayón-Ruisánchez et al. (2016) se trata de personas solidarias, preocupadas por cuidar y proteger el medioambiente, la naturaleza y los animales y que tienen un estilo de vida saludable e intentan ayudar en la medida de lo posible a los más necesitados.

Tabla 3: Principales países compradores de Comercio Justo del Mundo en 2015 (Miles de USD)

\begin{tabular}{|l|l|l|l|l|l|}
\hline País & Compra & País & Compra & País & Compra \\
\hline Alemania & 1.087 .032 & Estados Unidos & 1.018 .973 & Letonia & 961 \\
\hline Australia/N. Zelanda & 242.143 & Estonia & 3.619 & Lituania & 827 \\
\hline Austria & 205.625 & Finlandia & 192.872 & Luxemburgo & 12.004 \\
\hline Bélgica & 127.821 & Francia & 491.664 & Noruega & 89.558 \\
\hline Brasil & 67 & Hong Kong & 3.056 & Países Bajos & 247.861 \\
\hline Canadá & 302.934 & India & 841 & Reino Unido & 2.437 .847 \\
\hline Corea del Sur & 5.393 & Irlanda & 279.059 & República Checa & 9.512 \\
\hline Dinamarca & 113.841 & Italia & 110.070 & Sudáfrica & 20.603 \\
\hline Eslovaquia & 1.454 & Japón & 82.484 & Suecia & 387.352 \\
\hline España/Portugal & 31.241 & Kenia & 73 & Suiza & 528.257 \\
\hline
\end{tabular}

El proceso de determinar los vectores de ponderación para la priorización de los criterios para cada alternativa se muestran en la Tabla 4. Los pesos de cada criterio, subcriterios y características se establecieron a través del método de comparaciones pareadas y la escala de Saaty, cuya serie numérica de prioridades se presenta en la Tabla 5. Cabe señalar que se fijó como criterio de finalización del proceso de discusión de los resultados, el acuerdo del grupo de expertos para todos los criterios, subcriterios y características del modelo. Con todo, en la Tabla 6 se resume la matriz de comparación de criterios respecto al objetivo general usando una escala de proporciones. Finalmente en la Tabla 7 se ilustra la estructura del modelo jerárquico utilizado para medir el nivel de valoración para la priorización de los mercados internacionales que operan bajo Comercio Justo. De este modo, se genera una estructura anidada donde el resultado global, depende del resultado en los criterios y estos, por su parte, dependen del resultado de los subcriterios que los componen y así sucesivamente. 
Tabla 4: Esquema general de Análisis Jerárquico Multicriterio

\begin{tabular}{|c|c|c|c|c|c|c|}
\hline Objetivo & Criterios & Pesos & Subcriterios & Pesos & \multicolumn{2}{|c|}{ Alternativas (países con Comercio Justo) } \\
\hline \multirow{9}{*}{$\begin{array}{l}\text { Seleccionar o } \\
\text { Priorizar } \\
\text { Mercados } \\
\text { Internacionales }\end{array}$} & \multirow{5}{*}{$\begin{array}{c}1 . \\
\text { Criterio de } \\
\text { valoración } \\
\text { potencial del } \\
\text { mercado }\end{array}$} & \multirow{5}{*}{$W_{1}$} & Crecimiento Económico & $W_{11}$ & \multirow{9}{*}{$\begin{array}{c}\text { Alemania } \\
\text { Australia } \\
\text { Austria } \\
\text { Bélgica } \\
\text { Brasil } \\
\text { Canadá } \\
\text { Corea del Sur } \\
\text { Dinamarca } \\
\text { Eslovaquia } \\
\text { España } \\
\text { Estados Unidos } \\
\text { Estonia } \\
\text { Finlandia } \\
\text { Francia }\end{array}$} & \multirow{9}{*}{$\begin{array}{l}\text { Holanda } \\
\text { Hong Kong } \\
\text { Irlanda } \\
\text { Italia } \\
\text { Letonia } \\
\text { Lituania } \\
\text { Luxemburgo } \\
\text { Noruega } \\
\text { Nueva Zelanda } \\
\text { Portugal } \\
\text { Reino Unido } \\
\text { República Checa } \\
\text { Suecia } \\
\text { Suiza }\end{array}$} \\
\hline & & & Poder adquisitivo & $W_{12}$ & & \\
\hline & & & Volumen de importaciones & $W_{13}$ & & \\
\hline & & & Crecimiento de las importaciones & $W_{14}$ & & \\
\hline & & & Exportaciones & $W_{15}$ & & \\
\hline & \multirow{4}{*}{$\begin{array}{l}2 . \\
\text { Criterios para } \\
\text { valorar la } \\
\text { accesibilidad y } \\
\text { riesgo }\end{array}$} & \multirow{4}{*}{$\mathrm{W}_{2}$} & Barreras al comercio & $W_{21}$ & & \\
\hline & & & Riesgo & $W_{22}$ & & \\
\hline & & & Facilidad para hacer negocios & $\mathrm{W}_{23}$ & & \\
\hline & & & Transparencia y corrupción & $W_{24}$ & & \\
\hline
\end{tabular}

Tabla 5: Escala de Saaty

\begin{tabular}{|c|l|}
\hline Escala & Explicación de la escala verbal \\
\hline 1 & Ambos elementos poseen la misma importancia, por tanto, contribuyen de igual forma con la propiedad. \\
\hline 3 & Moderada importancia de un elemento sobre otro, por tanto, se favorece a un elemento por sobre otro. \\
\hline 5 & Fuerte importancia de un elemento sobre otro, por tanto, un elemento es fuertemente favorecido. \\
\hline 7 & Muy fuerte importancia de un elemento sobre otro, por tanto, un elemento es muy fuertemente dominante. \\
\hline 9 & $\begin{array}{l}\text { Extrema importancia de un elemento sobre otro, por tanto, un elemento es favorecido por lo menos con } \\
\text { un orden de magnitud de diferencia. }\end{array}$ \\
\hline
\end{tabular}

Tabla 6: Matriz de comparación de criterios respecto al objetivo general

\begin{tabular}{|c|c|c|}
\hline Priorización de Mercados Internacionales & $\begin{array}{c}\text { Criterio de valoración de } \\
\text { potencial de mercado }\end{array}$ & $\begin{array}{c}\text { Criterio para valorar la } \\
\text { accesibilidad y riesgo }\end{array}$ \\
\hline Criterio de valoración de potencial de mercado & 1 & $1 / 5$ \\
\hline Criterio para valorar la accesibilidad y riesgo & 5 & 1 \\
\hline Total & 6 & 1,2 \\
\hline
\end{tabular}

Tabla 7: Pesos de los criterios y subcriterios.

\begin{tabular}{|c|c|c|c|c|}
\hline Objetivo & Criterios & Pesos & Subcriterios & Pesos \\
\hline \multirow{9}{*}{$\begin{array}{l}\text { Seleccionar o } \\
\text { Priorizar Mercados } \\
\text { Internacionales }\end{array}$} & \multirow{5}{*}{$\begin{array}{l}\text { Criterios de valoración } \\
\text { potencial del mercado }\end{array}$} & \multirow{5}{*}{0,33} & Crecimiento Económico & 0,09 \\
\hline & & & Poder adquisitivo & 0,17 \\
\hline & & & Volumen de importaciones & 0,18 \\
\hline & & & Crecimiento de las importaciones & 0,10 \\
\hline & & & Exportaciones & 0,46 \\
\hline & \multirow{4}{*}{$\begin{array}{l}\text { Criterios para valorar la } \\
\text { accesibilidad y riesgo }\end{array}$} & \multirow{4}{*}{0,67} & Barreras al comercio & 0,49 \\
\hline & & & Riesgo & 0,08 \\
\hline & & & Facilidad para hacer negocios & 0,20 \\
\hline & & & Transparencia y corrupción & 0,23 \\
\hline
\end{tabular}

\section{RESULTADOS Y DISCUSIÓN}

En la Tabla 8 se detallan los pesos finales de los factores de vectores de ponderación para la jerarquización de los subcriterios bajo estudio, ordenados de mayor a menor importancia. Es así como las barreras al comercio $(32,8 \%)$, la transparencia-corrupción $(15,4 \%)$, las exportaciones $(15,2 \%)$ y la facilidad para hacer negocios (13,4\%\%), muestran ser los factores de mayor importancia relativa, sumando en total el $77 \%$ de las preferencias.

En sentido contrario, las variables con menor peso relativo son el crecimiento económico (3,0\%), el crecimiento de las importaciones (3,3\%), el riesgo $(5,4 \%)$, el poder adquisitivo $(5,6 \%)$ y el volumen de importaciones (5,9\%). 
Tabla 8: Vectores de ponderación para la jerarquización

\begin{tabular}{|l|c|}
\hline Factores & Vector de Ponderación \\
\hline Barreras al comercio & 0,3283 \\
\hline Transparencia y corrupción & 0,1541 \\
\hline Exportaciones & 0,1518 \\
\hline Facilidad para hacer negocios & 0,1340 \\
\hline Volumen importaciones & 0,0594 \\
\hline Poder adquisitivo & 0,0561 \\
\hline Riesgo & 0,0536 \\
\hline Crecimiento de las importaciones & 0,0330 \\
\hline Crecimiento Económico & 0,0297 \\
\hline
\end{tabular}

En el primer nivel de jerarquía (potencial de mercado), el criterio valorado con la mayor importancia son las exportaciones con un porcentaje del $15,2 \%$, seguido por volumen de importaciones con un 5,9\%; poder adquisitivo con 5,6\%; crecimiento de las importaciones con 3,3\% y finalmente crecimiento económico con el $3,0 \%$. Lo anterior permite evidenciar que, el aspecto más significativo, según los expertos, corresponde a características intrínsecas de la economía de origen, concretamente la capacidad exportadora de la Región Coquimbo, más que a las características extrínsecas del atractivo de mercado foráneo que opera bajo Comercio Justo, tales como el crecimiento de las importaciones o el crecimiento económico o el poder adquisitivo de sus habitantes. En el segundo nivel de jerarquía (accesibilidad y riesgo), 3 de los 4 subcriterios, obtuvieron las mayores puntuaciones de todas las variables (9) de estudio, representando el $61,6 \%$ del global. El criterio valorado con la mayor importancia es barreras al comercio con un porcentaje del $32,8 \%$, seguido por transparencia y corrupción con un 15,4\%; facilidad para hacer negocios con $13,4 \%$ y por último riesgo país con el $5,4 \%$.

De lo anterior llama especialmente la atención la relevancia otorgada a las barreras al comercio, pues diversos autores coinciden que mayores barreras a la exportación pueden afectar incluso el compromiso exportador (Al-Aali et al., 2012; Westhead et al., 2002; Yannopoulos y Kefalaki, 2010). De este modo entre mayores barreras encuentre una empresa para exportar, su gestión hacia el compromiso por desarrollar la actividad exportadora se verá restringido, la incertidumbre y el riesgo asumidos se acrecientan y es muy posible que las empresas opten por suspender el desarrollo de una estrategia de internacionalización (Marcela et al., 2013). Por tanto, los resultados obtenidos deberían estimular a los empresarios a invertir en mejorar sus recursos y capacidades asignadas a la actividad exportadora, como forma de enfrentar aquellas restricciones que pueden afectar negativamente su desempeño en el mercado internacional.

Al extraer los indicadores de las respectivas fuentes de información señaladas, es posible aplicar los vectores de ponderación para la jerarquización, apreciando que las mayores oportunidades para el desarrollo del Comercio Justo están concentradas en mayor medida en Estados Unidos y en los 15 países europeos, los que son identificados, precisamente, como los principales actores de la red Comercio Justo en el mundo (Ceccon Rocha y Ceccon, 2010). Específicamente, tal como lo estipula la Tabla 9, la revisión de los valores de priorización por continente o área geográfica, permite determinar que los mercados con mayor potencial de desarrollo según la matriz productiva de la IV Región se localizan en América del Norte (Estados Unidos, Canadá), Europa (Noruega, Dinamarca, Alemania y Suiza, entre otros) y Oceanía (Nueva Zelanda y Australia). Además, si se considera el comercio total desarrollado por Chile (exportaciones no minerales más importaciones), resulta que son justamente Estados Unidos (47\%) y Europa (40\%) quienes, en conjunto, representan casi el $90 \%$ del comercio total. Por su parte, Corea del Sur (en Asia) y Brasil (en América del Sur), se presentan como destinos potencialmente atractivos.

Tabla 9: Priorización de mercados internacionales por país y continente

\begin{tabular}{|c|c|c|c|}
\hline Continente & Ranking & País & Valor Ponderado \\
\hline \multirow{3}{*}{ América } & 1 & Estados Unidos & 0,167 \\
\cline { 2 - 4 } & 2 & Canadá & 0,086 \\
\cline { 2 - 4 } & 3 & Brasil & 0,054 \\
\hline \multirow{3}{*}{ Asia } & 1 & Corea del Sur & 0,058 \\
\cline { 2 - 4 } & 2 & Hong Kong & 0,051 \\
\hline \multirow{5}{*}{ Europa } & 1 & Noruega & 0,085 \\
\cline { 2 - 4 } & 2 & Dinamarca & 0,083 \\
\cline { 2 - 4 } & 3 & Alemania & 0,081 \\
\cline { 2 - 4 } & 4 & Suiza & 0,078 \\
\cline { 2 - 4 } & 5 & Suecia & 0,077 \\
\hline
\end{tabular}


Tabla 9: (continuación)

\begin{tabular}{|c|c|c|c|}
\hline Continente & Ranking & País & Valor Ponderado \\
\hline \multirow{5}{*}{ Europa } & 7 & Holanda & 0,067 \\
\cline { 2 - 4 } & 8 & España & 0,058 \\
\cline { 2 - 4 } & 9 & Francia & 0,058 \\
\cline { 2 - 4 } & 10 & Austria & 0,054 \\
\cline { 2 - 4 } & 11 & Bélgica & 0,053 \\
\cline { 2 - 4 } & 12 & Reino Unido & 0,053 \\
\cline { 2 - 4 } & 13 & Estonia & 0,052 \\
\cline { 2 - 4 } & 14 & Finlandia & 0,052 \\
\cline { 2 - 4 } & 15 & Letonia & 0,047 \\
\cline { 2 - 4 } & 16 & Lituania & 0,047 \\
\cline { 2 - 4 } & 18 & Italia & 0,047 \\
\cline { 2 - 4 } & 19 & República Checa & 0,044 \\
\cline { 2 - 4 } & 20 & Irlanda & 0,043 \\
\hline \multirow{5}{*}{ Oceanía } & 21 & Eslovaquia & 0,040 \\
\cline { 2 - 4 } & 2 & Portugal & 0,034 \\
\hline
\end{tabular}

Por último, cabe denotar la preocupación existente de diversos autores (Moore, 2004; Parvathi y Waibel, 2016; Rashid y Byun, 2018) en relación a que los productores asociados a Comercio Justo reporten, además de beneficios ecológicos y sociales, un desarrollo y bienestar económico efectivo, especialmente cuando se trata de micro y pequeñas empresas, caracterizadas por contar con un limitado flujo de efectivo y difícil acceso a fuentes de financiamiento (García y Villafuerte, 2015). Para ello, es fundamental la formulación de estrategias comerciales de exportación adaptadas al perfil de los países importadores de Comercio Justo, y alineadas a la realidad de los pequeños productores locales.

En tal sentido, la implementación de plataformas virtuales de Comercio Justo, podría convertirse en una opción interesante para estimular la asociatividad y cooperación entre productores. Tal como indican Sanabria et al. (2016) el comercio electrónico brinda a las MiPyMEs (micro, pequeñas y medianas empresas) diferentes beneficios, entre los que se cuentan: expansión del mercado, productividad e innovación, rentabilidad, incremento en las ventas y competitividad. Todos atributos requeridos para un proceso de internacionalización exitoso. Por lo demás, es necesario explorar nuevas alternativas de diversificación comercial, evaluando opciones como la agricultura orgánica combinada con los sistemas de comercialización de Comercio Justo, cuya integración podría generar un fortalecimiento mutuo y traer rendimientos conjuntos a los pequeños agricultores de economías emergentes (Parvathi y Waibel, 2013).

Asimismo cabe destacar lo mencionado por Villacrés et al. (2018) en cuanto a la importancia del trabajo colectivo entre el sector público y privado, pues si bien el Comercio Justo comprende un factor clave que contribuye a reducir la pobreza, esta será realmente efectiva si forma parte relevante de los planes de desarrollo de las administraciones locales, donde además las organizaciones gremiales realicen un apoyo real a las necesidades de los productores, y estos, a su vez, participen de manera activa exigiendo sus derechos y cumpliendo con las obligaciones comprometidas. De este modo podría impulsarse un proceso de desarrollo integral que además de la búsqueda de rentas monetarias (riqueza económica), se enfoque en derribar las diferentes privaciones que limitan el desarrollo pleno de las potencialidades y capacidades de sus participantes, dando así un mayor sentido a la noción de la pobreza como un fenómeno ineludiblemente multidimensional (Ferullo, 2006).

\section{CONCLUSIONES}

En base al análisis de los resultados del estudio se extraen tres conclusiones principales: 1) desde el punto de vista metodológico se confirma la fortaleza del Método AHP para la priorización de mercados y la posibilidad de replicarlo en contextos afines. No obstante se debe considerar que dichas adaptaciones deben ser realizadas por un grupo de expertos, con cualidades analíticas idóneas y que tengan amplios conocimientos del servicio requerido; 2) de acuerdo a los niveles de jerarquía analizados los criterios con mayor importancia relativa, para la priorización de mercados consumidores de Comercio Justo, son el volumen de exportaciones (dentro del potencial de mercado) y las barreras al comercio, transparencia \& corrupción y facilidad para hacer negocios (dentro de accesibilidad y riesgo); y 3) en cuanto a los hallazgos se revela que las mayores oportunidades de internacionalización para los productos de la región de Coquimbo se encuentran en América del Norte (principalmente Estados Unidos) y Europa, dejando a los países de Asia y Oceanía como potencialmente atractivos. 


\section{REFERENCIAS}

Al-Aali, A., J.S. Lim y H. Al-Aali, Perceived Export Barrier Differences Among Exporters: Less Developed Economy Evidence, doi: 10.5897/AJBM11.917, Afr. J. Bus. Manage., 6(36), 9945-9956 (2012)

Brunelli, M., Introduction to the Analytic Hierarchy Process, Springer, New York, USA (2015)

Cayón-Ruisánchez, E., M. García-de los Salmones y A. Pérez, El Perfil del Consumidor de Comercio Justo, Revista de Dirección y Administración de Empresas, ISSN: 2341-0108, (23), 75-95 (2016)

Ceccon Rocha, B. y E. Ceccon, La Red del Comercio Justo y sus Principales Actores, Invest. Geog., ISSN: 0188-4611, (71), 88-101 (2010)

Chávez, C. y V. Patraca, El Comercio Justo y la Responsabilidad Social Empresarial. Reflexiones desde los sistemas de certificación autónoma, Argumentos, ISSN: 0187-5795, 65, 229-259 (2011)

Comet, D., Comercio Justo: Una Alternativa Comercial Sostenible y Respetuosa con los Derechos Humanos, Revista de Antropologia Experimental, ISSN: 1578-4282, 16(6), 71-78 (2016)

Ferullo, H., El Concepto de Pobreza en Amartya Sen, Revista Valores en la Sociedad Industrial, ISSN: 0326-3398, 66, 10$16(2006)$

Garza, A., El Impacto del Comercio Justo en el Desarrollo de los Productores de Café, Estudios Sociales, ISSN: 0188 4557, 22(43), 272-293 (2014)

García, G. y M. Villafuerte, Las Restricciones al Financiamiento de las PYMES del Ecuador y su Incidencia de Política de Inversiones, Actualidad Contable Faces, ISSN: 1316-8533, 18(30), 49-73 (2015)

Hopkins, R., Impact Assessment Study of Oxfam Fair Trade, $1^{\text {a }}$ ed. Oxfam Fair Trade Programme, Oxford, Inglaterra (2000)

Jovanović, B., J. Filipović y V. Bakić, Prioritization of Manufacturing Sectors in Serbia for Energy Management Improvement - AHP method, doi: 10.1016/j.enconman.2015.03.107, Energy Convers. Manag., 98, $225-235$ (2015)

Llopis-Going, R., Consumo Responsable y Globalización Reflexiva. Un Estudio Referido al Comercio en España, Revista Española del Tercer Sector, ISSN: 1886-0400, 11, 145-165 (2009)

Marcela, D., A. Hurtado y M. Castillo, Influencia de las Barreras a la Exportación sobre el Compromiso Exportador y su incidencia en los Resultados Internacionales, doi: 10.21158/01208160.n75.2013.770, Revista Escuela de Administración de Negocios, (75), 38-55 (2013)

Marquina, P. y E. Reficco, Impacto de la Responsabilidad Social Empresarial en el Comportamiento de Compra y Disposición a Pagar de Consumidores Bogotanos, doi: 10.1016/j.estger.2015.10.001, Estud. Gerenc., 31(137), $373-382$ (2015)

Moore, G., The Fair Trade Movement : Parameters, Issues and Future Research, Journal of Business Ethics, ISSN: 15730697, 53(1), 73-86 (2004)

Oddershede, A., A. Arias y H. Cancino, Rural Development Decision Support Using the Analytic Hierarchy Process, doi: 10.1016/j.mcm.2007.03.006, Mathematical and Computer Modelling, 46(2007), 1107-1114 (2007)

Osorio, J., D. Manotas y L. Rivera, Priorización de Riesgos Operacionales para un Proveedor de Tercera Parte Logística - 3PL, doi: 10.4067/S0718-07642017000400016, Inf. Tecnol., 28(4), 135-144 (2017)

Parvathi, P. y H. Waibel, Fair Trade and Organic Agriculture in Developing Countries: A Review. doi: 10.1080/08974438.2013.736043, J. Int. Food Agribus. Mark., 25(4), 311-323 (2013)

Parvathi, P. y H. Waibel, Organic Agriculture and Fair Trade: A Happy Marriage? A Case Study of Certified Smallholder Black Pepper Farmers in India, doi: 10.1016/j.worlddev.2015.08.027, World Development, 77, 206-220 (2016)

Proqualitas, Identificación y Caracterización del Sector Comercio Justo y Consumo Responsable, Ministerio de Economía, Fomento y Turismo, Santiago de Chile (2015)

Rashid, M.S. y S.E. Byun, Are Consumers Willing to Go the Extra Mile for Fair Trade Products Made in a Developing Country? A Comparison with Made in USA Products at Different Prices, doi: 10.1016/j.jretconser.2017.12.011, Journal of Retailing and Consumer Services, 41(August 2017), 201-210 2018)

Raynolds, L.T., Fair Trade: Social Regulation in Global Food Markets, doi: 10.1016/j.jrurstud.2012.03.004, J. Rural Stud., 28(3), 276-287 (2012)

Saaty, T.L. y L.G. Vargas, Models, Methods, Concepts \& Applications of the Analytic Hierarchy Process, $2^{a}$ Ed., Springer, New York, USA (2012)

Sanabria, V., L. Torres y L. López, Comercio Electrónico y Nivel de Ventas en las Mipymes del Sector Comercio, Industria y Servicios de Ibagué, doi: 10.2106/JBJS.L.00314, Revista Escuela de Administración de Negocios, (80), 132-154 (2016)

Ulusoy, E., Experiential Responsible Consumption, doi: 10.1016/j.jbusres.2015.07.041, Journal of Business Research, 69(1), 284-297 (2016)

Villacrés, R., M. Bonilla, S. Fierro y M. Escobar, El Comercio Justo: Factor Clave de Disminución de la Pobreza en la Provincia Los Ríos, Ecuador, doi: 10.19044/esj.2018.v14n9p382, European Scientific Journal, 14(9), 1857-7881 (2018) 
Westhead, P., M. Wright y D. Ucbasaran, International Market Selection Strategies Selected by "Micro" and "Small" Firms, doi: 10.1007/s00520-005-0882-8, Omega, 30(2002), 51-68 (2002)

Yañez, L., Región de Coquimbo: Información Regional 2018, Oficina de Estudios y Políticas Agrarias (Odepa), Santiago de Chile, Chile (2018)

Yannopoulos, P. y M. Kefalaki, Export Barriers Facing Canadian SMEs: The Role of Mental Models, Journal of Business and Policy Research, ISSN: 1838-3742, 5(2), 54-68 (2010)

Wollmann, D., M.T.A. Steiner, G.E. Vieira y P.A. Steiner, Details of the Analytic Hierarchy Process Technique for the Evaluation of Health Insurance Companies, doi: 10.1590/S0103-65132013005000070, Production, 24(3), 583-593 (2014) 